\title{
Time-Domain Dynamics and Stability Analysis of Optoelectronic Oscillators Based on Whispering-Gallery Mode Resonators
}

\author{
Aurélien Coillet, Rémi Henriet, Patrice Salzenstein, Kien Phan Huy, Laurent Larger, Senior Member, IEEE, \\ and Yanne K. Chembo, Senior Member, IEEE
}

\begin{abstract}
Optoelectronic oscillators (OEOs) are microwave photonics systems intended to generate ultrastable radio-frequency signals with unprecedented phase noise performance for aerospace and communication engineering applications. They had originally been introduced in a configuration where the energy storage element was a fiber delay line. However, recent research in view of size and power consumption optimization has led to novel configurations where this fiber delay line is replaced by an ultrahigh $\mathrm{Q}$ whispering-gallery mode (WGM) resonator. So far, there has been no theoretical framework enabling to understand the dynamical behavior of these new architectures of OEOs. In this paper, we propose for the first time a deterministic time-domain model to investigate the dynamics of these OEOs based on WGM resonators. This model enables us to perform the stability analysis of the microwave oscillations, and to determine rigorously their range of stability as the loop gain is varied. After building the model, we perform a full stability analysis of the various stationary solutions for the microwave output. We then perform extensive numerical simulations, which are in complete agreement with the stability analysis. The theoretical analysis is also found to be in excellent agreement with our experimental measurements.
\end{abstract}

Index Terms-Microwave generation, nonlinear oscillators, optoelectronic devices.

\section{INTRODUCTION}

T HE optoelectronic oscillator (OEO) is nowadays considered as one of the most promising ultrastable microwave generator for applications in time-frequency metrology, frequency synthesis, detection, and navigation systems [1]. The first architecture of OEO, proposed by Yao and Maleki [2]-[4], performed energy storage in the feedback loop by using a few kilometer-long fiber-delay line instead of a high-finesse radiofrequency $(\mathrm{RF})$ filter. The idea to store laser light energy instead of microwave energy was a conceptual breakthrough which provided a technological pathway toward improved stability for RF

Manuscript received November 28, 2012; revised January 30, 2013; accepted March 1, 2013. This work was supported in part by the Centre National d'Etudes Spatiales under Project SHYRO (Action R\&T-R-S10/LN-0001004/DA:10076201), in part by the ANR project ORA (BLAN 031202), in part by the Région de Franche-Comté, and in part by the European Research Council under Project NextPhase (ERC StG 278616).

The authors are with the Optics Department, Franche Comté Electronique Mécanique Thermique et Optique-Sciences et Technologies, 25030 Besançon Cedex, France (e-mail: aurelien.coillet@femto-st.fr; remi.henriet@femto-st.fr; patrice.salzenstein@femto-st.fr; kphanhuy@univ-fcomte.fr; laurent.larger@ univ-fcomte.fr; yanne.chembo@femto-st.fr).

Color versions of one or more of the figures in this paper are available online at http://ieeexplore.ieee.org.

Digital Object Identifier 10.1109/JSTQE.2013.2252152 signal generators. Another noteworthy feature of OEOs is their nearly absolute frequency versatility, since the microwave can be arbitrarily set at any frequency belonging to the band $0.1-100$ GHz. The upper limit of this frequency band is in fact imposed by currently available optoelectronics components, and nothing theoretically prevents OEOs to generate millimeter waves as well.

Despite their excellent stability performances, the original fiber-based OEOs unfortunately have the disadvantage to be bulky because of the temperature-stabilized box containing the optical fiber delay line. Effectively, these fiber-based OEOs are not very transportable, and their weight might affect negatively the payload of spacecrafts. Their size (few $\mathrm{dm}^{3}$ ) also raises problems related to temperature stabilization, which becomes overly energy-greedy in this case. In addition, despite active stabilization, the long fiber delay line also induces an unavoidable phase drift that deteriorates the long-term stability of the oscillator. The ring-cavity modes induced by the fiber delay line are also arising as very strong (even though narrow) parasite peaks close to the carrier in the phase noise spectrum. These spurious peaks are indeed very detrimental in most applications.

In order to improve the features of this oscillator, many novel architectures have been proposed in recent years. Some of them involve multiple loops in order to suppress the spurious peaks [5], [6]. Others lock the oscillator to atomic resonances [7], or enhance the functionalities of the optical branch to improve the phase noise figure [8], the tunability of the oscillator [9], or to mode-lock the optical modes [10], [11] for ultralow jitter pulse generation.

However, one of the most interesting architecture to overcome the shortcomings of fiber-based OEOs is undoubtedly the configuration which replaces the fiber delay-line by an ultrahigh $Q$ WGM resonator (see, e.g., [12]-[15]). Whispering gallery mode (WGM) resonators are low-loss dielectric disks or rings that perform optical energy storage through trapping photons by total internal reflection [16], [17]. The optical $Q$ factor of these resonators can be defined as $Q=\omega_{0} / \Delta \omega$, where $\omega_{0}$ is the central angular frequency of the mode of interest and $\Delta \omega$ is the corresponding linewidth. When the resonators are almost perfectly shaped (with subnanometer surface roughness) with an ultralow-loss bulk material (fused silica, calcium, or magnesium fluoride crystals, etc.), they can achieve a quality factor that is typically above $10^{8}$ at $1550 \mathrm{~nm}$; they can even exceptionally reach record values higher than $10^{11}$ [18]. The linewidth $\Delta \omega=\omega_{0} / Q$ of these WGMs is typically of the order 
of $100 \mathrm{kHz}$ for $Q \sim 10^{9}$ at $1550 \mathrm{~nm}$. These linewidths are at least two orders of magnitude narrower than typical RF filters. Alternatively, the optical storage properties of WGM resonators can also be understood in terms of photon lifetime $\tau_{\mathrm{ph}}=Q / \omega_{0}$, which is of the order of $1 \mu \mathrm{s}$ for $Q \sim 10^{9}$ at $1550 \mathrm{~nm}$.

The modal structure of these resonators is such that their eigenmodes are grouped within families where WGMs are nearly equidistant when dispersion is neglected. For WGMs belonging to the same family, the intermodal frequency (which is sometimes referred to as free spectral range, or FSR) is a free parameter that depends on the resonator's principal radius. It may vary from $\mathrm{GHz}$ (millimeter-size disks) to $\mathrm{THz}$ (micrometer-size) frequencies. Therefore, in the case of OEOs based on WGM resonators, we can obtain a microwave oscillation by extracting the intermodal frequency of an optically pumped WGM resonator, while the energy storage would be performed by trapping photons in the long-lifetime WGM cavity. This architecture solves almost all the problems raised by fiber-based OEOs, and yields an oscillator that is versatile, compact, energy efficient, and free from parasite spurious peaks (see, e.g., [19]). Most importantly, it is expected that these WGM-based architectures would enable us to downsize the OEO from a shoe box (fiber-based OEO) to a notebook (WGM-based OEO), and ultimately, to a smartphone (integrated WGM-based OEO), without deteriorating the phase noise performance in the most favorable case.

Even though it appears clearly that WGM-based OEOs will play an increasingly important role in microwave photonics, there is currently no dynamical framework to study their dynamics. This lack of analytical insight into the dynamical properties of this oscillator does not enable us to optimize its properties. In particular, it is impossible to know under which conditions the oscillator is merely stable, and it is worth reminding that this stability problem is far from being trivial.

Our aim in this paper is, therefore, to propose a deterministic model to understand and analyze the time-domain dynamics of WGM-based OEOs. Using this model, we will study the stability of the various solutions, and perform numerical simulations to confirm the stability analysis study. We have also built a new architecture of WGM-based OEO, and performed experimental measurements to check the validity of the model. Both the theoretical and experimental studies agree with excellent precision, thereby confirming the validity of the nonlinear dynamics framework of analysis.

This paper is organized as follows. The next section is devoted to the description of the experimental system under study, which is an architecture of WGM-based OEO with amplitude modulation and add-drop coupling. In Section III, we present the main lines of our theoretical approach for OEOs, which is based on the nonlinear dynamics of the slowly varying microwave envelope, while Section IV is focused on the construction of the theoretical model for this WGM-based OEO. Then, we determine the stationary states of the oscillator in Section V, while their stability is investigated in Sections VI and VII. Hence, we perform numerical simulations that are compared to experimental measurements in Section VIII. The last section concludes this paper with a resume of our work and perspectives of future research.

\section{EXPERIMENTAL SYSTEM}

The WGM-based OEO under study is displayed in Fig. 1. The various elements of this single-loop architecture are as follows.

1) A continuous-wave (CW) distributed feedback (DFB) semiconductor laser of optical power $P_{L}$ and whose central wavelength is $\lambda_{L}=1552.2 \mathrm{~nm}$, corresponding to an angular frequency $\omega_{L}=2 \pi c / \lambda_{L}$, where $c$ is the velocity of light in vacuum.

2) An erbium-doped fiber amplifier (EDFA) delivering a maximal optical power of $30 \mathrm{dBm}$, and of optical gain $G_{o}$ when driven by the input laser.

3) A wideband integrated optics $\mathrm{LiNbO}_{3}$ Mach-Zehnder (MZ) intensity modulator characterized by the half-wave voltages $V_{\pi_{\mathrm{DC}}}=4 \mathrm{~V}$ and $V_{\pi_{\mathrm{RF}}}=4.7 \mathrm{~V}$.

4) A polarization controller to tune the polarization at the input of the MZ intensity modulator and the WGM resonator.

5) A crystalline calcium fluoride $\left(\mathrm{CaF}_{2}\right)$ WGM resonator coupled in the add-drop configuration. The intrinsic, excitation, and drop $Q$ factors are, respectively, $Q_{i}, Q_{e}$, and $Q_{d}$. They, respectively, correspond to photon lifetimes $Q_{i, e, d} / \omega_{L}=\tau_{i, e, d} / 2$, where $\omega_{L}$ is the angular frequency of the laser while the $\tau$ parameters are the laser field decay times. The internal quality factor $Q_{i}$ is fixed and equal to $4 \times 10^{8}$, while the coupling quality factors $Q_{e}$ and $Q_{d}$ can be varied by changing the position of the coupling tapered fibers relatively to the resonator; in all case, these coupling $Q$-factors are of the order of $10^{8}$. The WGM disk has a refractive index equal to $n_{g}=1.43$ and a principal radius $a=3.2 \mathrm{~mm}$. In consequence, its free spectral range is a microwave frequency equal to $\Omega_{M}=c / a n_{g}=2 \pi \times 10.4 \mathrm{GHz}$.

6) A fast photodiode with a conversion factor $S=50 \Omega \times$ $0.75 \mathrm{~A} / \mathrm{W}=37.5 \mathrm{~V} / \mathrm{W}$, and bandwidth $0-12 \mathrm{GHz}$.

7) A narrowband microwave RF filter of central frequency at $10.5 \mathrm{GHz}$ and a bandwidth of $1 \mathrm{GHz}$, intended to reject the RF noise outside the frequency band of interest.

8) Cascaded microwave amplifiers with overall gain $G_{e}$ are used to close the loop and drive the intensity modulator.

The principle of operation of this WGM-based OEO is, therefore, the following: noise in the microwave branch of the loop modulates a laser light beam, and generates a broadband optical spectrum at the output of the MZ modulator. This broadband, initially noisy optical spectrum, is narrowly filtered by the WGM resonator. At the output, the optical spectrum is now a set of equidistant spectral lines, separated by the FSR of the resonator (that is, $\simeq 10 \mathrm{GHz}$ ). The photodiode detects the various beating frequencies $k \Omega_{M}$ ( $k$ being an integer), but because of its limited bandwidth, it filters them out except the fundamental frequency $\Omega_{M}$ and the dc component of the field. The RF filter rejects this dc component. Then, the microwave signal at $\Omega_{M}$ is finally amplified and sent as a driving signal to the $\mathrm{MZ}$ in order to close the feedback loop and the same cycle is started again.

It is known from oscillator engineering theory that the oscillation might be sustained when the Barkhausen conditions are fulfilled, that is, when 1) the loop gain overcomes the loop losses 


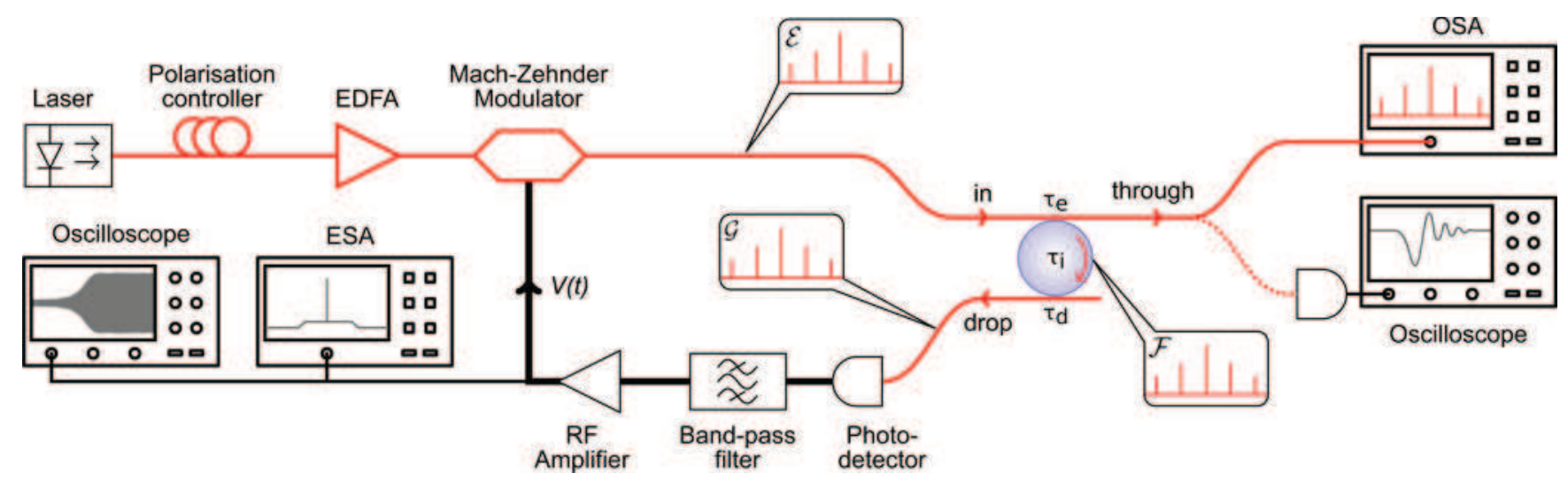

Fig. 1. Experimental setup. EDFA: erbium-doped fiber amplifier; ESA: electrical spectrum analyzer; OSA: optical spectrum analyzer. The optical path is in thin red, while the electric path is in thick black. Inserts show the spectra of the optical fields $\mathcal{E}(t), \mathcal{F}(t)$, and $\mathcal{G}(t)$ at the input, interior, and output of the WGM resonator, respectively. The spectral lines are separated by the frequency $\Omega_{M}$ which corresponds to the FSR of the resonator. The "through" port of the coupling fibers is used to monitor the optical spectra with the OSA, in both the transient and stationary regimes. In the electric branch, a fast oscilloscope enables us to resolve the temporal dynamics of the microwave $V(t)$, whose complex envelope is $\mathcal{V}(t)$ (or $\mathcal{A}(t)$ in the dimensionless form). An ESA also enables us to monitor the corresponding RF spectrum. In the open-loop configuration (no oscillations), the "through" port is also used to perform the cavity-ring down measurement using an oscilloscope, thereby enabling the determination of the intrinsic and in-coupling quality factors. An optical attenuator (nor represented) has been inserted between the EDFA and the MZ in order to control the incoming optical power.

and 2) the round-trip phase of the microwave signal is null modulo $2 \pi$. However, the Barkhausen theory cannot describe what might occur above threshold (amplitude of the oscillations, multistability, hysteresis, higher order bifurcations, chaos, etc.), nor does it enable the stability study of the oscillating solutions. The purpose of the nonlinear dynamics approach is to shed the light on all these blind spots, as it provides a complete understanding of the oscillator behavior below and above threshold. We will explain in the next section how this nonlinear dynamics is used in the context of OEOs in general, and WGM-based OEOs in particular.

\section{Modeling OEOs: A MicrowaVe EnVElope APPROACH}

A microwave oscillator whose output angular frequency is around $\Omega_{0}$ is always expected to have an output of the form

$$
V(t)=A(t) \cos \left[\Omega_{0} t+\psi(t)\right]
$$

where $A(t)$ and $\psi(t)$ are, respectively, the amplitude and the phase of the microwave. In the case where $A$ and $\psi$ are constant, the microwave output $V(t)$ is perfectly sinusoidal. The microwave is still sinusoidal as well if $\psi=\psi_{0}+\sigma t$, even though the oscillation frequency is shifted to $\Omega_{0}+\sigma$. In the general case, both $A$ and $\psi$ will be time dependent, but their time variation will be significantly slower than the period of the oscillation. More concretely, $A(t)$ and $\psi(t)$ will vary at a slow time scale comparable to the inverse RF bandwidth $1 / \Delta \Omega$ of the oscillation loop, while the full microwave $V(t)$ will vary at a fast time scale of $1 / \Omega_{0}$. Therefore, the slow and fast dynamics are split by a factor $Q_{\mathrm{RF}}=\Omega_{0} / \Delta \Omega$ corresponding to the RF quality factor of the oscillator. This is why $A(t)$ and $\psi(t)$ are referred to as slowly varying amplitude and slowly varying phase, respectively.

In general, the only variables of interest are $A$ and $\psi$ since $\Omega_{0}$ is known. Equation (1) can be rewritten under the useful form

$$
V(t)=\frac{1}{2} \mathcal{V}(t) e^{i \Omega_{0} t}+\frac{1}{2} \mathcal{V}^{*}(t) e^{-i \Omega_{0} t}
$$

where $\mathcal{V}(t)=A(t) e^{i \psi(t)}$, and the star denotes the complex conjugation. Here, the complex-valued variable $\mathcal{V}(t)$ synthetically gathers all the information about the amplitude and the phase of the microwave: it is referred to as the complex slowly varying envelope of the microwave. Hence, it is the idoneous variable to investigate the dynamics of the RF output of a microwave oscillator. It is interesting to note that complex slowly varying envelopes are routinely used in optics and laser theory, where the nominal frequencies (from the lasers, optical cavities, etc.) are generally known, while the optical amplitudes and phases are the variables of interest. It is also worth noting that the complex envelope takes into account eventual frequency detunings $\sigma$ from the nominal frequency $\Omega_{0}$ through $\psi(t)$.

The microwave envelope approach has already been used with great success in fiber-based OEOs. In fact, fiber-based OEOs belong to the large family of electro-optic systems with delayed feedback, which can be described by Ikeda-like delaydifferential equations where the main variable is the real-valued voltage at the input of the modulator (see [20]). In the particular case of fiber-based OEOs, the narrowband filtering around the frequency $\Omega_{0}$ of interest enables us to rewrite this voltage under the form of (2). A nonlinear delay-differential equation ruling the dynamics of the complex microwave envelope had, therefore, been obtained, thereby enabling to demonstrate many fundamental results. Just to name a few, this nonlinear dynamics approach enables us to prove that fiber-based OEOs can turn unstable if the feedback gain exceeds a precise bifurcation value [21], [22]. The same approach also enabled us to show that under certain conditions, the abrupt switch-on behavior of the fiber-based OEOs leads to robust multimode oscillations instead of an ultrastable single-tone microwave [23]. The timedomain deterministic model was also an essential prerequisite that enabled us to perform a phase noise analysis based on stochastic differential equations (or Langevin equations) and which enabled us to predict phase noise characteristics with remarkable precision [24], [25]. The same formalism also enabled 
us to analyze more complex OEO architectures, like dual-loop OEOs [26], or hybrid configurations whose outputs are an ultrastable microwave in the RF domain and an ultralow jitter picosecond pulse train in the optical domain [27].

We show in the next section that a complex envelope formalism can be developed as well for WGM-based OEOs.

\section{MODEL}

The dynamics of this system is essentially defined by two variables. The first variable is the laser electric field $E(t)$ at the input of the resonator and the second variable is the input microwave voltage $V(t)$ of the integrated modulator.

Instead of working directly with the real-valued $E(t)$ and $V(t)$, we use their complex slowly varying amplitudes $\mathcal{E}(t)=$ $|\mathcal{E}(t)| e^{i \varphi(t)}$ and $\mathcal{V}(t)=|\mathcal{V}(t)| e^{i \psi(t)}$ defined through

$$
\begin{aligned}
E(t) & =\frac{1}{2} \mathcal{E}(t) e^{i \omega_{L} t}+\frac{1}{2} \mathcal{E}^{*}(t) e^{-i \omega_{L} t} \\
V(t) & =\frac{1}{2} \mathcal{V}(t) e^{i \Omega_{M} t}+\frac{1}{2} \mathcal{V}^{*}(t) e^{-i \Omega_{M} t}
\end{aligned}
$$

where $\omega_{L}$ and $\Omega_{M}$ are the angular frequencies associated with the $1550 \mathrm{~nm}$ infrared laser beam, and with the $10 \mathrm{GHz}$ microwave signal, respectively.

The slowly varying amplitude of the optical beam at the input of the MZ modulator simply reads $\mathcal{E}_{\mathrm{cw}}=\sqrt{P_{0}}$, where $P_{0}=$ $G_{o} P_{L}$ is the optical power at the output of the EDFA. This pumping field sets the optical phase reference, and as a consequence is real (its phase is null). This beam is amplitude-modulated with a driving RF signal $V(t)=|\mathcal{V}(t)| \cos \left[\Omega_{M} t+\psi(t)\right]$, so that the slowly varying amplitude of the optical field at the input of the optical fiber is

$$
\begin{aligned}
\mathcal{E}(t) & =\mathcal{E}_{\mathrm{cW}} \cos \left\{\frac{\pi V}{2 V_{\pi_{\mathrm{RF}}}}+\frac{\pi V_{B}(t)}{2 V_{\pi_{\mathrm{DC}}}}\right\} \\
& =\sqrt{P_{0}} \cos \left\{\frac{\pi|\mathcal{V}(t)|}{2 V_{\pi_{\mathrm{RF}}}} \cos \left[\Omega_{M} t+\psi(t)\right]+\frac{\pi V_{B}}{2 V_{\pi_{\mathrm{DC}}}}\right\} .
\end{aligned}
$$

The Jacobi-Anger expansion gives

$$
e^{i z \cos \alpha}=\sum_{n=-\infty}^{+\infty} i^{n} \mathrm{~J}_{n}(z) e^{i n \alpha}
$$

where $\mathrm{J}_{n}$ is the $n$ th-order Bessel function of the first kind. Therefore, we have

$$
\mathcal{E}(t)=\sqrt{P_{0}} \sum_{n=-\infty}^{+\infty} \mathcal{E}_{n}(t) e^{i n \Omega_{M} t}
$$

where the dimensionless modal fields are

$$
\mathcal{E}_{n}(t)=\epsilon_{n}(\phi) \mathrm{J}_{n}\left[\frac{\pi|\mathcal{V}(t)|}{2 V_{\pi_{R F}}}\right] e^{i n \psi(t)}
$$

with

$$
\begin{aligned}
\epsilon_{n}(\phi) & =\frac{1}{2}\left[e^{i \phi}+(-1)^{n} e^{-i \phi}\right] i^{n} \\
& = \begin{cases}(-1)^{\frac{n}{2}} \cos \phi, & \text { if } n \text { is even } \\
(-1)^{\frac{n+1}{2}} \sin \phi, & \text { if } n \text { is odd } .\end{cases}
\end{aligned}
$$

The parameter

$$
\phi=\frac{\pi V_{B}}{2 V_{\pi_{D C}}}
$$

is the offset phase due to the bias voltage of the integrated MZ modulator. The intracavity field $\mathcal{F}(t)$ inside the resonator can also be spectrally decomposed as $\mathcal{F}(t)=$ $\sum_{n=-\infty}^{+\infty} \mathcal{F}_{n}(t) e^{i n \Omega_{M} t}$. According to the Haus formalism [28], the dimensionless components $\mathcal{F}_{n}$ obey

$$
\frac{\mathrm{d} \mathcal{F}_{n}}{\mathrm{~d} t}=-\frac{1}{\tau} \mathcal{F}_{n}-i \sigma \mathcal{F}_{n}+\sqrt{\frac{2}{\tau_{e}}} \mathcal{E}_{n}
$$

where $\tau$ is defined as

$$
\frac{1}{\tau}=\frac{1}{\tau_{i}}+\frac{1}{\tau_{e}}+\frac{1}{\tau_{d}}
$$

and stands for the overall loss-induced decay time for the electric fields inside the resonator, while $\sigma=\omega_{L}-\omega_{0}$ is the laser detuning relatively to the central frequency of the pumped mode. On the other hand, the dimensionless components $\mathcal{G}_{n}$ of the output field $\mathcal{G}(t)=\sum_{n=-\infty}^{+\infty} \mathcal{G}_{n}(t) e^{i n \Omega_{M} t}$ can simply be recovered as

$$
\mathcal{G}_{n}=\sqrt{\frac{2}{\tau_{d}}} \mathcal{F}_{n} .
$$

The spectra of the three optical fields $\mathcal{E}(t), \mathcal{F}(t)$, and $\mathcal{G}(t)$ have been schematically represented in Fig. 1.

The optical power at the input of the photodiode is equal to

$$
\begin{aligned}
P_{0}|\mathcal{G}(t)|^{2} & =P_{0}\left|\sum_{n=-\infty}^{+\infty} \mathcal{G}_{n}(t) e^{i n \Omega_{M} t}\right|^{2} \\
& =\frac{1}{2} \mathcal{C}_{0}(t)+\sum_{k=1}^{+\infty}\left\{\frac{1}{2} \mathcal{C}_{k}(t) e^{i k \Omega_{M} t}+\text { c.c. }\right\}
\end{aligned}
$$

where the slowly varying Fourier coefficients $\mathcal{C}_{k}$ express the multifrequency nature of the input optical power. The photodiode has an inbuilt filter that rejects the harmonics at $k \Omega_{M}$ with $k \geq 2$. On the other hand, the dc component is rejected by the RF bandpass filter. Hence, only the spectral component at frequency $\Omega_{M}$ (that is, $\mathcal{C}_{ \pm 1}(t)$ ) is allowed to pass through. The slowly varying amplitude $\mathcal{V}$ of the voltage at the output of the photodiode is, therefore, $S \mathcal{C}_{1}(t)$, where $S$ stands for the photodiode sensitivity. We can multiply this output by the overall gain $G=G_{e} G_{o}$ and the overall losses $\kappa$ to obtain the slowly varying voltage at the input of the modulator as

$$
\mathcal{V}(t)=\kappa G S e^{i \varsigma} \mathcal{C}_{1}(t)=\kappa G S P_{0} \sum_{n=-\infty}^{+\infty} 2 \mathcal{G}_{n+1} \mathcal{G}_{n}^{*}
$$

where the phase factor $e^{i \varsigma}$ accounts for the effect of the microwave round-trip phase shift $\varsigma$. If necessary, it can be tuned to any desired value (modulo $2 \pi$ ) using an RF phase shifter.

We define the dimensionless microwave voltage as

$$
\mathcal{A}(t)=\frac{\pi}{2 V_{\pi_{\mathrm{RF}}}} \mathcal{V}(t)
$$


and the optoelectronic gain as

$$
\beta=\frac{\pi \kappa G S P_{0}}{2 V_{\pi_{\mathrm{RF}}}} .
$$

It is very important to note that since the loss parameter $\kappa$ does not consider the losses associated with the WGM resonator, the gain coefficient $\beta$ is not a loop-gain parameter as it is the case for fiber-based OEO studies, where $\kappa$ also takes into account the losses induced by the fiber.

We, therefore, have the following three-step model for numerical simulation:

$$
\begin{aligned}
& \mathcal{E}_{n}=\epsilon_{n}(\phi) \mathrm{Jc}_{n}[|\mathcal{A}|] \mathcal{A}^{n} \\
& \dot{\mathcal{G}_{n}}=-\left[\frac{1}{\tau}+i \sigma\right] \mathcal{G}_{n}+\frac{2}{\sqrt{\tau_{e} \tau_{d}}} \mathcal{E}_{n} \\
& \mathcal{A}=2 \beta e^{i \varsigma} \sum_{n=-\infty}^{+\infty} \mathcal{G}_{n+1} \mathcal{G}_{n}^{*}
\end{aligned}
$$

where the overdot stands for time derivative. Note that in (17), we introduced the Bessel cardinal functions to simplify the expression. Their definition and properties are further developed in the Appendix.

This model describes the following phenomenology. Small noise in $\mathcal{A}$ generates the fields $\mathcal{E}_{n}$, which are fed in the WGM resonator and yield the output fields $\mathcal{G}_{n}$. The photodiode extracts the intermodal frequency and the bandpass RF filter outputs a microwave of complex envelope $\mathcal{A}$, which is plugged back to the modulator to generate new fields $\mathcal{E}_{n}$, and the previous sequence of events takes place again.

It is interesting to note that fiber-based OEOs have an optical delay line that performs the optical energy storage, and an RF filter to select the microwave oscillation frequency. On the other hand, WGM-based OEOs rather have a WGM disk that performs at the same time the optical storage and filtering functions. The physics is intrinsically different, and so are the corresponding models: this explains why the former models built for fiberbased OEOs are not valid anymore in this context. Fiber-based OEO models relied on delay-differential equations, with the delay being induced by the fiber delay line, and the dynamics (i.e., the derivative term) was on the microwave variable $\mathcal{A}(t)$. For WGM-based OEOs, we rather have a modal expansion model, in the sense that we have one equation per optical mode, and the dynamics is on the optical modes $\mathcal{G}_{n}(t)$. It is also important to note that this model is nonlinear and continuous. Hence, we can analytically and numerically determine the various dynamical behaviors of the system, and investigate their stability.

\section{STATIONARY STATES}

The equilibria (or fixed points) of autonomous oscillators are obtained by setting all the derivatives to zero. In the complex envelope formalism, a trivial equilibrium corresponds to the absence of oscillation, while nontrivial equilibria correspond to a steady-state oscillation (because the amplitudes are constant and not null). The aim of this section is to determine the fixed points of the OEO.
The stationarity conditions $\dot{\mathcal{G}_{n}} \equiv 0$ yield

$$
\mathcal{G}_{n}=\mathcal{T} \mathcal{E}_{n}
$$

where

$$
\mathcal{T}=\frac{\frac{2}{\sqrt{\tau_{e} \tau_{d}}}}{\frac{1}{\tau}+i \sigma}
$$

is the transmission coefficient of the WGM resonator at the drop port. It is interesting to note that we always have $|\mathcal{T}| \leq 1$, and ideal transmission $(\mathcal{T}=1)$ only occurs when a resonant laser radiation $(\sigma=0)$ is coupled to a lossless resonator $\left(\tau_{i} \rightarrow+\infty\right)$ with coupling photon lifetimes that are matched $\left(\tau_{e}=\tau_{d}\right)$.

According to (19) and (20), we have

$$
\mathcal{A}_{\mathrm{st}}=2 \beta e^{i \varsigma} \sum_{n=-\infty}^{+\infty} \mathcal{G}_{n+1} \mathcal{G}_{n}^{*}=2 \beta e^{i \varsigma}|\mathcal{T}|^{2} \sum_{n=-\infty}^{+\infty} \mathcal{E}_{n+1} \mathcal{E}_{n}^{*}
$$

Hence, using (17), the stationary amplitude $A_{\text {st }}=\left|\mathcal{A}_{\text {st }}\right|$ obeys

$$
A_{\mathrm{st}}=-\beta \sin 2 \phi|\mathcal{T}|^{2} e^{i \varsigma} \sum_{n=-\infty}^{+\infty}(-1)^{n} \mathrm{~J}_{n+1}\left(A_{\mathrm{st}}\right) \mathrm{J}_{n}\left(A_{\mathrm{st}}\right)
$$

and using successively the Bessel equalities

$$
\begin{aligned}
\mathrm{J}_{-n}(x) & =(-1)^{n} \mathrm{~J}_{n}(x) \\
\mathrm{J}_{m}(x+y) & =\sum_{n=-\infty}^{+\infty} \mathrm{J}_{n}(x) \mathrm{J}_{m-n}(y)
\end{aligned}
$$

we are finally led to the transcendental equation

$$
A_{\mathrm{st}}=\Gamma \mathrm{J}_{1}\left(2 A_{\mathrm{st}}\right)
$$

where

$$
\Gamma=-\beta \sin 2 \phi|\mathcal{T}|^{2} e^{i \varsigma}
$$

is the overall loop gain, which is essentially the product of the optoelectronic gain and the power transmission factor of the coupled WGM resonator. Existence of a stationary state requires the phase factor $e^{i \varsigma}$ to be real, and equal to \pm 1 such that $\Gamma$ is real and positive. Effectively, this round-trip phase matching condition corresponds to the necessity of a constructive interference between successive round-trip replicas of the microwave (Barkhausen condition for the phase).

The possible solutions of the transcendental equation (26) are, therefore,

$$
A_{\mathrm{st}}= \begin{cases}A_{\mathrm{tr}}=0, & \text { valid for all } \Gamma \\ A_{\mathrm{osc}}=\frac{1}{2} \mathrm{Jc}_{1}^{-1}\left[\frac{1}{2 \Gamma}\right], & \text { valid for all } \Gamma \geq 1\end{cases}
$$

where $\mathrm{Jc}_{1}^{-1}$ is the inverse Bessel-Cardinal function. The trivial equilibrium $A_{\mathrm{tr}}$ is, therefore, a solution for all values of $\Gamma$, while nontrivial (oscillatory) solutions $A_{\text {osc }}$ can only exist for $\Gamma \geq 1$.

As explained in Fig. 2, the stationary solutions of the transcendental equation (26) are given by the intersection of the functions $\mathrm{J}_{1}\left(2 A_{\mathrm{st}}\right)$ and $A_{\mathrm{st}} / \Gamma$. When $\Gamma<1$, there is only one solution (the trivial one), while for $\Gamma>1$, both functions will intersect for other points than 0 , thereby generating nontrivial equilibria. When $1<\Gamma<15.52$, there is only one oscillatory 


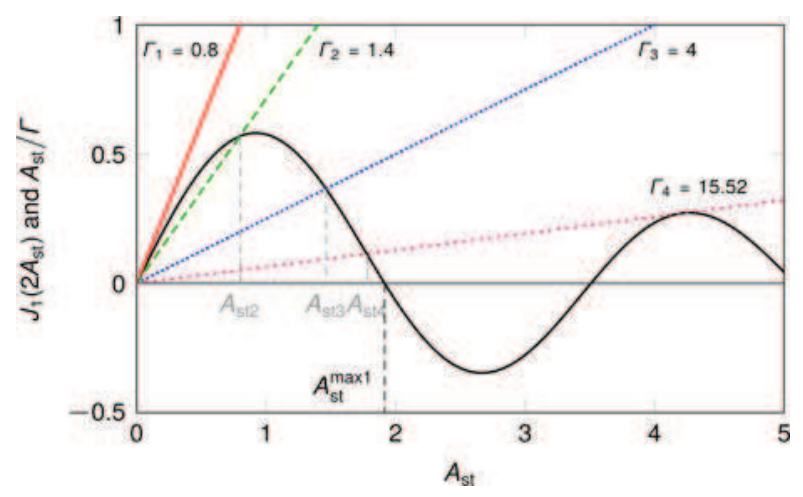

Fig. 2. Geometrical interpretation of the stationary states $A_{\text {st }}$, which are given by the intersection of the functions $\mathrm{J}_{1}\left(2 A_{\mathrm{st}}\right)$ and $A_{\text {st }} / \Gamma$. When $\Gamma=0.8$, both curves only intersect for $A_{\mathrm{st}}=0$, which is, therefore, the unique fixed point. This will be the case whenever $\Gamma<1$. For $\Gamma>1$, both functions will intersect for other points than 0 , thereby generating nontrivial equilibria. For $\Gamma_{2}=1.4$, the nontrivial solution is $A_{\mathrm{st} 2}=0.79$, and it will be equal to $A_{\mathrm{st} 3}=1.46$ for $\Gamma_{3}=4$. When the gain is increased to $\Gamma_{4}=15.52$, the same branch yields a nontrivial solution $A_{\mathrm{st} 4}=1.77$, while a pair of new solutions are created around 4.21. An infinity of other pairs of solutions are sequentially created as $\Gamma$ increases to infinity.

solution. However, at $\Gamma=15.52$, a new pair of nontrivial solutions emerges and coexists with the previous oscillatory state. As $\Gamma$ increases to infinity, an infinity of branches generating paired solutions are created; it appears very clearly that all of them are converging to the zeros of the Bessel function $\mathrm{J}_{1}(2 x)$ when $\Gamma \rightarrow+\infty$.

It is important to note that realistic values for the normalized gain $\Gamma$ are generally not as high as 15 . That is why in all the experiments of OEOs, the oscillator is generally operated in the first branch of nontrivial solutions, whose maximal value is the first zero of $\mathrm{J}_{1}\left(2 A_{\mathrm{st}}\right)$, yielding $A_{\mathrm{st}}^{\max 1}=1.91$. This asymptotic saturation can be observed in Fig. 3. In this paper, we will refer to this branch of nontrivial solution as the primary branch of oscillatory solutions. Fig. 3 also displays the emergence of the secondary branch of paired solutions above $\Gamma=15.52$.

After the determination of the various stationary states of the system, we will perform in the next two sections their stability analysis.

\section{Stability of ThE TRIVIAL EQUilibrium}

The onset of oscillations generally occurs when the trivial fixed point $\mathcal{A}_{\mathrm{tr}}=0$ loses its stability. In this section, we perform the stability analysis of this fixed point in order to determine the conditions leading to oscillations.

Let us consider a perturbation $\delta \mathcal{A}$ around the trivial equilibrium $\mathcal{A}_{\mathrm{tr}}=0$. If that perturbation decreases with time, the trivial equilibrium is stable; otherwise, if it increases, the rest point is unstable and oscillations are triggered. The optical spectral components $\mathcal{E}_{n}$ excited by the perturbation $\delta \mathcal{A}$ explicitly read

$$
\mathcal{E}_{n} \simeq \epsilon_{n}(\phi) \times \begin{cases}\frac{1}{2^{n} n !}(\delta \mathcal{A})^{n}, & \text { if } n \geq 0 \\ \frac{(-1)^{-n}}{2^{-n}(-n) !}\left(\delta \mathcal{A}^{-n}\right)^{*}, & \text { if } n<0 .\end{cases}
$$

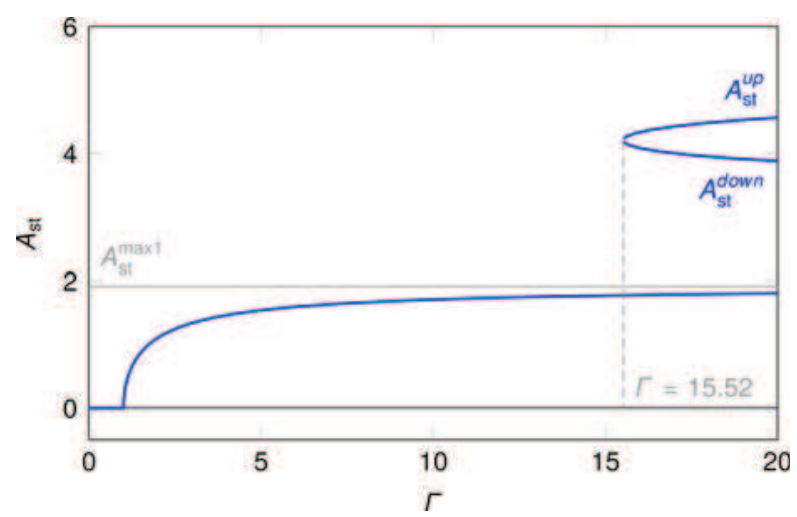

Fig. 3. Variation of the nontrivial solutions of (26) as $\Gamma$ is increased. Only the trivial solution exists for $\Gamma<1$. Then, the primary branch is the unique nontrivial solution for $1<\Gamma<15.52$. This primary branch of solutions, which exists for all $\Gamma>1$, increases monotonously but has a horizontal asymptote $A_{\mathrm{st}}^{\max 1}=1.91$. New nontrivial solutions (secondary branch) emerge for $\Gamma>$ 15.52. The bifurcation analysis shows that the microwave envelope $\mathcal{A}$ undergoes a pitchfork bifurcation at $\Gamma=1$, which corresponds to a Hopf bifurcation for the microwave voltage variable $V$. The same analysis also shows that $\mathcal{A}$ undergoes a saddle-node bifurcation at $\Gamma=15.52$ (emergence of two new fixed points, one being stable and the other one unstable). The system, therefore, starts in that case to evidence hysteresis, with two stable solutions (lowest and highest amplitudes) and an unstable solution (corresponding to the intermediate amplitude). As $\Gamma$ is further increased, the system undergoes saddle-node bifurcations any time a new branch of paired solutions emerges.

It appears that whenever $|n|>1$, the fields $\mathcal{E}_{n}$ are of higher order of perturbation, and can, therefore, be neglected in a linear stability analysis. On the other hand, the induced fields at orders $n=0, \pm 1$ are explicitly given by

$$
\begin{aligned}
\mathcal{E}_{1} & =-\frac{1}{2} \sin \phi \delta \mathcal{A} \\
\mathcal{E}_{0} & =\cos \phi \\
\mathcal{E}_{-1} & =-\frac{1}{2} \sin \phi \delta \mathcal{A}^{*} .
\end{aligned}
$$

In other words, the mode $n=0$ is of zeroth order and is not influenced by the microwave perturbation $\delta \mathcal{A}$ in the linear approximation, while the modes $n= \pm 1$ are of first order and are directly proportional to $|\delta \mathcal{A}|$. For the sake of mathematical clarity, we will then rewrite the fields $\mathcal{E}_{ \pm 1}$ as $\delta \mathcal{E}_{ \pm 1}$ since they are first-order perturbations. It straightforwardly appears that the modal output variables related to $\mathcal{G}$ will have the same order of magnitude as their input counterpart $\mathcal{E}$. Hence, we will have to consider only the variables $\mathcal{G}_{0}$ and $\delta \mathcal{G}_{ \pm 1}$, and neglect all the remaining ones.

Using (19), the microwave perturbation can be rewritten as

$$
\delta \mathcal{A}=2 \beta e^{i \varsigma}\left[\mathcal{G}_{0} \delta \mathcal{G}_{-1}^{*}+\mathcal{G}_{0}^{*} \delta \mathcal{G}_{1}\right]
$$

while according to (18), the output field perturbations $\mathcal{G}_{ \pm 1}$ obey

$$
\begin{aligned}
\delta \dot{\mathcal{G}}_{1} & =-\left[\frac{1}{\tau}+i \sigma\right] \delta \mathcal{G}_{1}+\frac{2}{\sqrt{\tau_{e} \tau_{d}}} \delta \mathcal{E}_{1} \\
\delta \dot{\mathcal{G}}_{-1} & =-\left[\frac{1}{\tau}+i \sigma\right] \delta \mathcal{G}_{-1}+\frac{2}{\sqrt{\tau_{e} \tau_{d}}} \delta \mathcal{E}_{-1}
\end{aligned}
$$


In the matrix form, the aforementioned equation can be rewritten as

$$
\left[\begin{array}{c}
\delta \dot{\mathcal{G}}_{1} \\
\delta \dot{\mathcal{G}}_{-1}^{*}
\end{array}\right]=\left[\mathbf{S}_{\mathrm{tr}}\right]\left[\begin{array}{c}
\delta \mathcal{G}_{1} \\
\delta \mathcal{G}_{-1}^{*}
\end{array}\right]
$$

where

$$
\left[\mathbf{S}_{\mathrm{tr}}\right]=\frac{2}{\sqrt{\tau_{e} \tau_{d}}}\left[\begin{array}{cc}
\frac{1}{\mathcal{T}}\left(\frac{\Gamma}{2}-1\right), & \frac{\Gamma}{2 \mathcal{T}^{*}} \\
\frac{\Gamma}{2 \mathcal{T}}, & \frac{1}{\mathcal{T}^{*}}\left(\frac{\Gamma}{2}-1\right)
\end{array}\right]
$$

is a $2 \times 2$ matrix ruling the dynamics of the perturbation flow (Jacobian). The trivial fixed point will be stable whenever the eigenvalues of this Jacobian matrix have strictly negative real parts. The determination of these eigenvalues is straightforward and it is found that the trivial equilibrium is stable only when $\Gamma<1$, and unstable otherwise.

We will show in the next section that crossing that threshold value triggers unconditionally stable microwave oscillations in the primary branch of nontrivial equilibria, while for the higher order branches of paired solutions, some solutions are stable while others are not.

\section{Stability OF THE OSCILlatory SOlution}

So far, the theoretical analysis has shown that there are two types of stationary solutions. The trivial equilibrium exists for all $\Gamma$ but is stable only for $\Gamma<1$. The oscillatory solutions only exists for $\Gamma>1$, and the purpose of this section is to demonstrate that they might be stable or unstable. Once again, to demonstrate that this solution is stable, we have to show that any perturbation $\delta \mathcal{A}$ of the oscillatory solution $A_{\mathrm{osc}}$ of interest exponentially decays to zero. Otherwise, the oscillatory solution is unstable.

According to (19), the perturbation of the steady state microwave solution $A_{\text {osc }}$ obeys

$$
\delta \mathcal{A}=2 \beta e^{i \varsigma} \sum_{n=-\infty}^{+\infty} \mathcal{G}_{n+1} \delta \mathcal{G}_{n}^{*}+\mathcal{G}_{n}^{*} \delta \mathcal{G}_{n+1} .
$$

On the other hand, the steady-state input and output electric fields obey

$$
\begin{aligned}
& \mathcal{E}_{n}=\epsilon_{n}(\phi) \mathrm{J}_{n}\left(A_{\mathrm{osc}}\right) \\
& \mathcal{G}_{n}=\mathcal{T} \mathcal{E}_{n} .
\end{aligned}
$$

In the demonstration, we have made to investigate the stability of the trivial fixed point, it appeared that the Jacobian matrix $\left[\mathbf{S}_{\mathrm{tr}}\right]$ had to be expressed relatively to the variables $\delta \mathcal{G}_{1}$ and $\delta \mathcal{G}_{-1}^{*}$. In the case of the nontrivial solutions, (38) shows that we have an infinity of perturbations to consider, but however, we will decompose by analogy the microwave perturbation as

$$
\delta \mathcal{A}=\delta \mathcal{A}_{+}+\delta \mathcal{A}
$$

where

$$
\begin{aligned}
& \delta \mathcal{A}_{+}=2 \beta e^{i \varsigma} \sum_{n=-\infty}^{+\infty} \mathcal{T}^{*} \mathcal{E}_{n-1}^{*} \delta \mathcal{G}_{n} \\
& \delta \mathcal{A}_{-}=2 \beta e^{i \varsigma} \sum_{n=-\infty}^{+\infty} \mathcal{T} \mathcal{E}_{1-n} \delta \mathcal{G}_{-n}^{*}
\end{aligned}
$$

are global variables associated to the output field perturbations $\delta \mathcal{G}_{n}$ and their counterparts $\delta \mathcal{G}_{-n}^{*}$ (complex conjugate, opposite sidemode), respectively. We will, hereafter, use these variables to obtain a Jacobian matrix whose eigenvalues will decide the stability of the nontrivial stationary states.

In the feedback loop, the perturbation $\delta \mathcal{A}$ will first induce perturbations $\delta \mathcal{E}_{n}$. The first-order Taylor expansion of a perturbation of the amplitude can be determined as

$$
\left|A_{\mathrm{osc}}+\delta \mathcal{A}\right| \simeq A_{\mathrm{osc}}+\frac{1}{2}\left[\delta \mathcal{A}+\delta \mathcal{A}^{*}\right]
$$

Hence, using (39), the input field perturbations can be calculated as

$$
\delta \mathcal{E}_{n}=\frac{1}{2} \epsilon_{n}(\phi) \mathrm{J}_{n}^{\prime}\left(A_{\mathrm{osc}}\right)\left[\delta \mathcal{A}+\delta \mathcal{A}^{*}\right]
$$

where the prime denotes the derivative of the Bessel function relatively to its argument $A_{\mathrm{osc}}$. The input field perturbations $\delta \mathcal{E}_{n}$ do induce output field perturbations $\delta \mathcal{G}_{n}$, which obey

$$
\delta \dot{\mathcal{G}_{n}}=-\left[\frac{1}{\tau}+i \sigma\right] \delta \mathcal{G}_{n}+\frac{2}{\sqrt{\tau_{e} \tau_{d}}} \delta \mathcal{E}_{n} .
$$

By multiplying the aforementioned equation by $2 \beta e^{i \varsigma} \mathcal{T}^{*} \mathcal{E}_{n-1}^{*}$ and summing over all modal indices $n$, we are led to the following equation for $\delta \mathcal{A}_{+}$

$$
\delta \dot{\mathcal{A}_{+}}=-\left[\frac{1}{\tau}+i \sigma\right] \delta \mathcal{A}_{+}+\frac{4 \beta e^{i \varsigma} \mathcal{T}^{*}}{\sqrt{\tau_{e} \tau_{d}}} \sum_{n=-\infty}^{+\infty} \mathcal{E}_{n-1}^{*} \delta \mathcal{E}_{n}
$$

and analogously, it can be found that $\delta \mathcal{A}_{-}$obeys

$$
\delta \dot{\mathcal{A}}_{-}=-\left[\frac{1}{\tau}-i \sigma\right] \delta \mathcal{A}_{-}+\frac{4 \beta e^{i \varsigma} \mathcal{T}}{\sqrt{\tau_{e} \tau_{d}}} \sum_{n=-\infty}^{+\infty} \mathcal{E}_{1-n} \delta \mathcal{E}_{-n}^{*}
$$

We demonstrate in the Appendix that the perturbation equations (47) and (48) can be rewritten as

$$
\begin{aligned}
& \delta \dot{\mathcal{A}}_{+}=-\left[\frac{1}{\tau}+i \sigma\right]\left\{\delta \mathcal{A}_{+}-R\left[\delta \mathcal{A}+\delta \mathcal{A}^{*}\right]\right\} \\
& \delta \dot{\mathcal{A}}_{-}=-\left[\frac{1}{\tau}-i \sigma\right]\left\{\delta \mathcal{A}_{-}-R\left[\delta \mathcal{A}+\delta \mathcal{A}^{*}\right]\right\}
\end{aligned}
$$

where $R$ is a function of the gain $\Gamma$ :

$$
R=\frac{1}{4} \Gamma\left[\mathrm{J}_{0}\left(2 A_{\mathrm{osc}}\right)-\mathrm{J}_{2}\left(2 A_{\mathrm{osc}}\right)\right]
$$

Since $\delta \mathcal{A}=\delta \mathcal{A}_{+}+\delta \mathcal{A}_{-}$, we can finally rewrite (49) and (50) under the form of a 4-D autonomous flow

$$
\left[\begin{array}{l}
\delta \dot{\mathcal{A}}_{+} \\
\delta \dot{\mathcal{A}}_{-} \\
\delta \dot{\mathcal{A}}_{+}^{*} \\
\delta \dot{\mathcal{A}}_{-}^{*}
\end{array}\right]=\left[\mathbf{S}_{\text {osc }}\right]\left[\begin{array}{l}
\delta \mathcal{A}_{+} \\
\delta \mathcal{A}_{-} \\
\delta \mathcal{A}_{+}^{*} \\
\delta \mathcal{A}_{-}^{*}
\end{array}\right]
$$

where the Jacobian matrix $\left[\mathbf{S}_{\mathbf{o s c}}\right]$ can be written under the synthetic block matrix form

$$
\left[\mathbf{S}_{\mathbf{o s c}}\right]=\left[\begin{array}{cc}
{[\mathbf{U}]} & {[\mathbf{V}]} \\
{\left[\mathbf{V}^{*}\right]} & {\left[\mathbf{U}^{*}\right]}
\end{array}\right]
$$




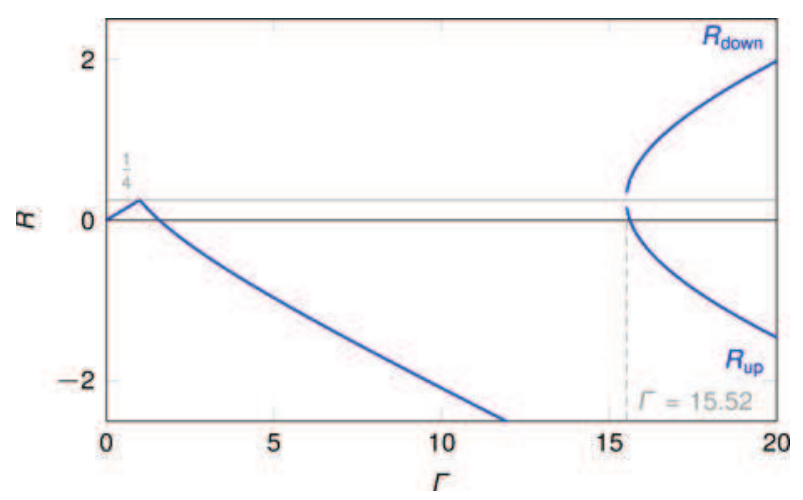

Fig. 4. Variations of the function $R$ expressed in (51). This function is inferior to $1 / 4$ for the primary branch of nontrivial solutions (starting at $\Gamma=1$ ). For the secondary branch (starting at $\Gamma=15.52)$, one solution is stable $(R<1 / 4)$ while the other one is unstable $(R>1 / 4)$.

with

$$
\begin{aligned}
& {[\mathbf{U}]=\frac{2}{\sqrt{\tau_{e} \tau_{d}}}\left[\begin{array}{cc}
\frac{R-1}{\mathcal{T}} & \frac{R}{\mathcal{T}} \\
\frac{R}{\mathcal{T}^{*}} & \frac{R-1}{\mathcal{T}^{*}}
\end{array}\right]} \\
& {[\mathbf{V}]=\frac{2}{\sqrt{\tau_{e} \tau_{d}}}\left[\begin{array}{ll}
\frac{R}{\mathcal{T}} & \frac{R}{\mathcal{T}} \\
\frac{R}{\mathcal{T}^{*}} & \frac{R}{\mathcal{T}^{*}}
\end{array}\right] .}
\end{aligned}
$$

The oscillatory solution $A_{\mathrm{osc}}$ is therefore stable if all the eigenvalues of the constant and complex-valued matrix $\left[\mathbf{S}_{\mathbf{o s c}}\right]$ have a strictly negative real part. A straightforward method would be to actually compute the eigenvalues of this 4-D matrix and evaluate the sign of their respective real parts. However, we can circumvent that tedious task by noting due to the particular structure of $\left[\mathbf{S}_{\mathbf{o s c}}\right]$, the set of its eigenvalues is the union of the eigenvalues of the 2-D matrices $[\mathbf{U}+\mathbf{V}]$ and $[\mathbf{U}-\mathbf{V}]$. Hence, the stability of the oscillations is guaranteed as long as the eigenvalues of the $2 \times 2$ matrices $[\mathbf{U} \pm \mathbf{V}]$ have strictly negative real parts. This stability condition is trivially satisfied for $[\mathbf{U}-\mathbf{V}]$ which is a diagonal matrix whose diagonal elements (i.e., eigenvalues) are $-1 / \tau \pm i \sigma$. On the other hand, as far as the matrix $[\mathbf{U}+\mathbf{V}]$ is concerned, the stability condition yields $R<\frac{1}{4}$. According to Fig. 4, this inequality is indeed satisfied whenever $\Gamma>1$ for the branch of primary solutions, since $R$ has an absolute maximum value equal to $\frac{1}{4}$ for $\Gamma=1$, and decreases monotonously afterward. On the other hand, the higher order branches of paired solutions have the typical stability pattern of saddle-node fixed points, as one solution remains stable while the other is unstable.

We recall again that experimentally, it is extremely difficult to reach $\Gamma$ values of the order of 15 . Most experimental studies can in fact hardly achieve gain values superior to 3 . Therefore, the solution of practical interest belongs to the primary branch: our analysis has demonstrated that this oscillating solution is unique in the range $1<\Gamma<15.52$, and is always exponentially stable for any $\Gamma>1$. It is interesting to note that in the case of fiber-based OEOs, we proved that the microwave oscillation was stable only at up to $\Gamma=2.31$, in agreement with experi-

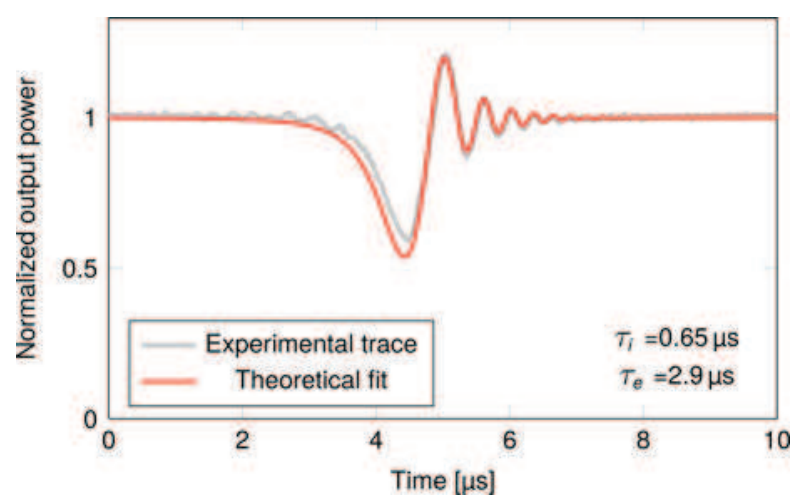

Fig. 5. Cavity ring-down measurement. The thick gray curve is the experimental optical power at the output of the "through" port of the resonator when the input wavelength is rapidly swept. The optical signal at resonance decays exponentially and interferes with the next wavelengths coming from the input laser. The theoretical fit that enables us to extract the intrinsic and excitation photon lifetimes is derived in [29]. Here, the intrinsic and excitation photon lifetimes are measured at 0.65 and $2.9 \mu \mathrm{s}$, respectively.

ments [21], [22]. Here, we prove that the oscillator is unconditionally stable at up to a much higher value (15.52), thereby demonstrating that this WGM-based OEO is significantly more stable than its fiber-based counterpart.

\section{COMPARISON BETWEEN NUMERICAL AND EXPERIMENTAL RESULTS}

The experimental setup is presented in detail in Fig. 1.

A preliminary measurement is the evaluation of the intrinsic and coupling $Q$ factors of the cavity. This measure, presented in Fig. 5 is performed in the open-loop configuration and is mathematically explained in [29]. It enables us to confirm that all our quality factors are of the order of $10^{8}$, yielding characteristic time scales of the order of $1 \mu \mathrm{s}$. When the oscillation loop is closed, we can monitor different optical and microwave variables of interest.

The optical output of the coupled resonator's through-port is used to control the detuning $\sigma$ between the input laser and the resonance. Once the detuning is set and the gain is above threshold, oscillations are sustained in the optical branch. Fig. 6 shows the optical spectrum taken at the "through" output of the resonator (as drawn in Fig. 1). The spectral line due to the laser is in the center at $f_{L}=193276.6 \mathrm{GHz}$, and two pairs of sidemodes are visible in this case, separated by the FSR $f_{M}=10.4 \mathrm{GHz}$. As we can see on this 75-dB dynamic-range figure, the amplitude of these sidemodes decreases very rapidly with their order $|n|$.

In the RF branch, the spectrum of the microwave oscillation is measured using a 22-GHz RF spectrum analyzer. A typical RF spectrum is presented in Fig. 7 and demonstrates that the oscillation arises at a fixed frequency given by the FSR $f_{M}$ of the resonator. A plateau is visible below the oscillation spectral line and is due to the noise of the RF amplifier filtered between 10 and $11 \mathrm{GHz}$.

Simultaneously, the temporal dynamics of the microwave signal is monitored using a 40-GHz-bandwidth oscilloscope. To investigate this time-domain dynamics, another $\mathrm{MZ}$ modulator 


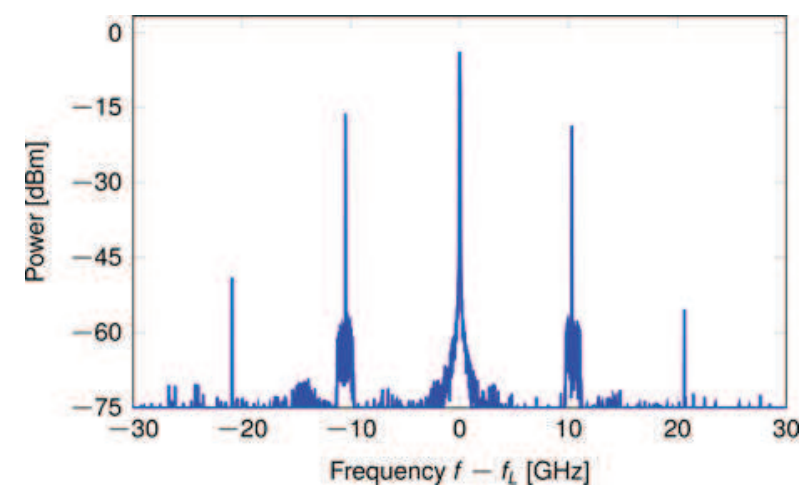

Fig. 6. Optical spectrum of the signal at the throughport of the resonator. The central frequency $f_{L}=193276.6 \mathrm{GHz}$ is the optical frequency of the input laser. The sidemodes created by the MZ intensity modulator driven by the microwave oscillation are clearly visible on each side of the input frequency. The microwave noise from the amplifier is visible as a plateau on each sidemode, on top of which the oscillation at the FSR frequency stands. It is apparent on this figure that the amplitudes of the sidemodes decrease very rapidly, so that only few of them are necessary to describe the behavior of the oscillator in the numerical simulations.

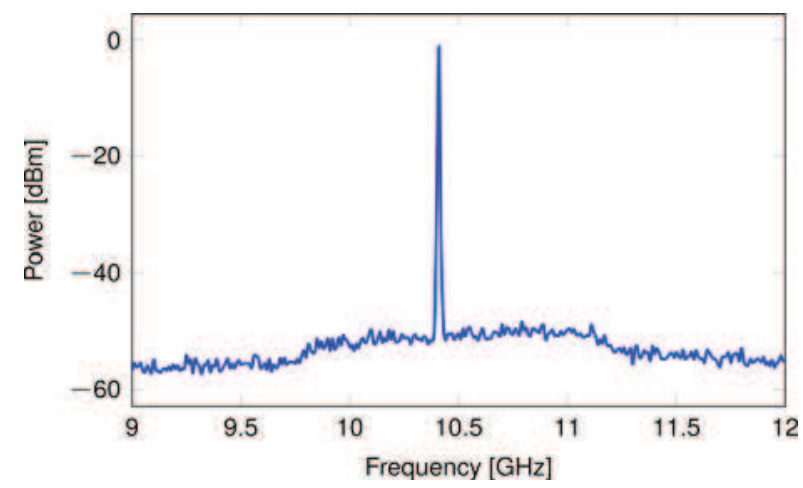

Fig. 7. RF spectrum of the generated microwave. The 10.41-GHz oscillation corresponding to the FSR of the resonator. This microwave signal is strong, and stands $50 \mathrm{~dB}$ above the filtered noise of the RF amplifier.

driven by a square signal is used to switch the input laser light $\mathrm{ON}$ and OFF. Therefore, the gain $\Gamma$ of the oscillator is abruptly changed from 0 to a value that is higher than 1 , and the transient dynamics can be monitored using a photodiode and a fast oscilloscope. The resulting signal is displayed in Fig. 8. The actual signal $V(t)$ is shown in light gray while the envelope amplitude $|\mathcal{V}(t)|$ is the thick blue curve. The inset of this figure is provided to show the oscillation occurring at a faster time scale (in the range of $100 \mathrm{ps}$, which corresponds to the period $T_{M}=1 / f_{M}$ ), while the envelope time scale is in the microsecond range. It is worth noting that the apparent amplitude noise in this inset is an artefact due to the barely sufficient sampling rate of the oscilloscope $(30 \mathrm{GHz})$ compared to the oscillation frequency (10 GHz).

The envelope curve of Fig. 8 can be compared to the numerical simulations presented in Fig. 9. These simulations were obtained using a fourth-order Runge-Kutta algorithm for (17)(19). The initial conditions where random complex value for $\mathcal{A}$, taken in a Gaussian distribution centered around 0.01 with standard deviation of 0.01 . Both numerical and experimental curves feature the same characteristics, and the transition from the ini-

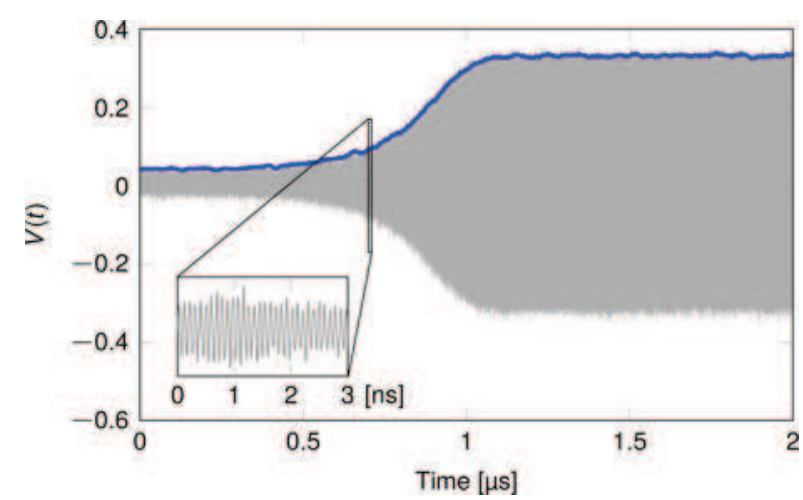

Fig. 8. Experimental time-domain dynamics. The (light gray) experimental trace of the microwave signal is obtained with an ultrafast oscilloscope, just after abruptly switching-on the laser. At the very beginning, the signal consists of noise from the RF amplifier. The RF oscillation at the FSR frequency rapidly grows above the noise level, and its amplitude reaches the stationary state with a time scale in the $\mu$ s range. The inset is a zoom-in presenting the fast-scale dynamics of the same curve. It shows that the microwave frequency is indeed around $10 \mathrm{GHz}$. The apparent amplitude noise is due on the one hand to the detection photodiode, and on the other to an artefact originating from the fact that albeit ultrafast, the $30 \mathrm{GHz}$ sampling rate only provides six points for every period of our $10 \mathrm{GHz}$ signal. The experimental microwave envelope signal (thick blue line) is in very good agreement with the normalized envelope $\mathcal{A}(t)$ obtained numerically like in Fig. 9.

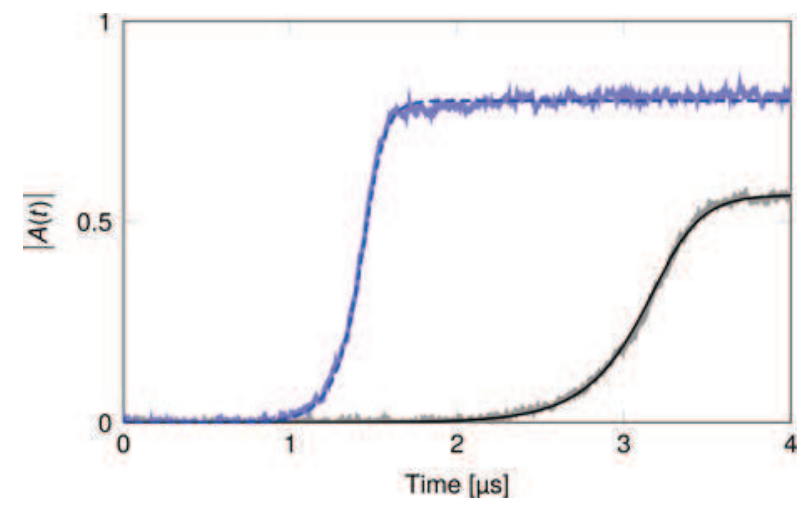

Fig. 9. Numerical simulation of the microwave envelope dynamics, and comparison with experiments. The continuous black (down) and dashed blue (top) curves are numerical simulations obtained for $\Gamma=1.18$ and 1.55 , respectively. The other parameters are $\tau_{i}=0.65 \mu \mathrm{s}, \tau_{e}=2.9 \mu \mathrm{s}$ (same as the experimental values), $\tau_{d}=0.1 \mu \mathrm{s}, \sigma=0$ (at resonance), and $\phi=\pi / 4$. The initial condition is a random value taken in a Gaussian distribution with average value and standard deviation of 0.01 . The envelopes of experimental time traces are plotted in thick lighter lines on top of the continuous black and dashed blue curves (the photodiode and artefact noise have been subtracted).

tial state to the stationary solution occurs in similar duration, of the order of $1 \mu \mathrm{s}$. This very good agreement between the simulation and the experimental result validates the experimental interest of this model.

It is interesting to note that from a purely theoretical point of view, the model is infinite dimensional because there is an infinity of fields $\mathcal{G}_{n}$ to consider. However, only a few of them are necessary to yield accurate results, as foreshadowed by the experimental spectrum of Fig. 6. In practice, our simulations were performed with ten pairs of sidemodes, even though considering five pairs would have already been very accurate. 


\section{IX, CONCLUSION}

In this paper, we have proposed a nonlinear dynamics approach to study WGM-Based OEOs. We have used a complex microwave envelope variable to investigate the time-domain behavior and stability properties of this oscillator. Our study has enabled us to determine the various stationary states and their stability. It was shown that above threshold, the principal branch of the oscillations, which is the only one experimentally accessible so far, is always exponentially stable regardless of the gain. However, the analysis has also evidenced higher order branches of solutions whose stability properties are more complex, with some states being stable while the others are not. Both the analytical and numerical analysis have been confirmed by the experimental measurements.

Future work will consist in using the model to optimize the metrics of the oscillator. In particular, we aim to investigate the phase noise performance of this WGM-based OEO by adding calibrated noise terms in our dynamical equations. We would then obtain stochastic differential equations that would enable us to predict the phase noise spectra and the Allan deviation of the oscillator.

It is already known that very high microwave frequencies, at the edge of the millimeter-waves band ( $\simeq 100 \mathrm{GHz}$ ), can be generated by selecting a higher harmonic of the beat-note signal detected by the photodetector. Our model enables us to analyze the microwave envelope of such harmonics $k \Omega_{M}$ by summing the quadratic terms $\mathcal{G}_{n+k} \mathcal{G}_{n}^{*}$ in (14).

Another prospective work would be to extend this formalism in order to account for nonlinear phenomena in the WGM resonator. Some research works (see e.g., [30]) have already demonstrated that scattering has a measurable effect on the phase noise performance of OEOs, particularly when the optical cavity has a high $Q$ factor. Various nonlinear phenomena (Kerr, Raman, and Brillouin) have an effect on the phase noise performance, as well as the chromatic dispersion which converts laser frequency noise into phase fluctuations. We expect this theoretical approach to be able to give both a quantitative and qualitative insight into all into these phenomenologies.

\section{APPENDIX}

\section{A. Bessel-Cardinal Functions}

We define the Bessel-cardinal function of order $n$ as

$$
\mathrm{Jc}_{n}(x)=\frac{\mathrm{J}_{n}(x)}{x^{n}} \text { with } x \in \mathbb{R} \text { and } n \in \mathbb{Z}
$$

where $\mathrm{J}_{n}$ is the $n$th order Bessel function of the first kind. From a qualitative point of view, Bessel-cardinal functions look like the sine-cardinal function $\operatorname{sinc}(x)=\sin x / x$ when $n>0$, with an absolute maximum centered at $x=0$, and an oscillatory behavior converging to zero as $x \rightarrow \pm \infty$. On the other hand, the Bessel-cardinal function diverges to infinity as $x \rightarrow \pm \infty$ with an oscillatory behavior when $n<0$. Since $\mathrm{J}_{n}(r) e^{i n \theta}$ can be rewitten under the analytical form $z^{n} \mathrm{Jc}_{n}(|z|)$ with $z=r e^{i \theta}$, the Bessel-cardinal formalism is very useful to carry out some of the mathematical calculations.

\section{B. Demonstration of (49) and (50)}

This demonstration relies on the explicit calculation of the infinite sums in the right-hand side of (47) and (48).

Let us first calculate the sum in (49). Using (39) and (44) and the recurrence relationship

$$
\mathrm{J}_{n}^{\prime}(x)=\frac{1}{2}\left[\mathrm{~J}_{n-1}(x)-\mathrm{J}_{n+1}(x)\right]
$$

we explicitly have

$$
\begin{aligned}
\sum_{n=-\infty}^{+\infty} \mathcal{E}_{n-1}^{*} \delta \mathcal{E}_{n}= & \frac{1}{4}\left[\delta \mathcal{A}+\delta \mathcal{A}^{*}\right] \\
& \times \sum_{n=-\infty}^{+\infty}\left\{\epsilon_{n}(\phi) \epsilon_{n-1}(\phi) \mathrm{J}_{n-1}\left(A_{\mathrm{osc}}\right)\right. \\
& \left.\times\left[\mathrm{J}_{n-1}\left(A_{\mathrm{osc}}\right)-\mathrm{J}_{n+1}\left(A_{\mathrm{osc}}\right)\right]\right\} .
\end{aligned}
$$

However, we have $\epsilon_{n}(\phi) \epsilon_{n-1}(\phi)=(-1)^{n} \sin \phi \cos \phi$, while (24) and (25) yield the following Bessel relationships

$$
\begin{aligned}
\sum_{n=-\infty}^{+\infty}(-1)^{n} \mathrm{~J}_{n-1}^{2}(x) & =-\sum_{n=-\infty}^{+\infty} \mathrm{J}_{1-n}(x) \mathrm{J}_{n-1}(x) \\
& =-\mathrm{J}_{0}(2 x) \\
\sum_{n=-\infty}^{+\infty}(-1)^{n} \mathrm{~J}_{n-1}(x) \mathrm{J}_{n+1}(x) & =-\sum_{n=-\infty}^{+\infty} \mathrm{J}_{1-n}(x) \mathrm{J}_{n+1}(x) \\
& =-\mathrm{J}_{2}(2 x) .
\end{aligned}
$$

Hence, (59) can be finally simplified to

$$
\begin{aligned}
\sum_{n=-\infty}^{+\infty} \mathcal{E}_{n-1}^{*} \delta \mathcal{E}_{n}=-\frac{1}{4} \sin \phi & \cos \phi\left(\delta \mathcal{A}+\delta \mathcal{A}^{*}\right) \\
& \times\left[\mathrm{J}_{0}\left(2 A_{\mathrm{osc}}\right)-\mathrm{J}_{2}\left(2 A_{\mathrm{osc}}\right)\right]
\end{aligned}
$$

Since $\epsilon_{-n}(\phi)=(-1)^{n} \epsilon_{n}(\phi)$, it can also be shown that in the nontrivial stationary states, $\mathcal{E}_{-n}^{*}=\mathcal{E}_{n}$ and $\delta \mathcal{E}_{-n}^{*}=\delta \mathcal{E}_{n}$, so that the infinite sums in the right-hand sides of (47) and (48) are identical. Then, using (27) and (61) the demonstration of (49) and (50) is straightforward.

\section{ACKNOWLEDGMENT}

The authors would like to thank P. Féron for insightful discussions about the theoretical and experimental characterization of WGM resonators.

\section{REFERENCES}

[1] L. Maleki, "The optoelectronic oscillator," Nature Photon., vol. 5, pp. 728730, 2011.

[2] X. S. Yao and L. Maleki, "High frequency optical subcarrier generator," Electron. Lett., vol. 30, pp. 1525-1526, 1994

[3] X. S. Yao and L. Maleki, "Optoelectronic microwave oscillator," J. Opt. Soc. Amer. B, vol. 13, pp. 1725-1735, 1996.

[4] X. S. Yao and L. Maleki, "Optoelectronic oscillator for photonic systems," IEEE J. Quantum Electron., vol. 32, no. 7, pp. 1141-1149, Jul. 1996.

[5] X. S. Yao and L. Maleki, "Dual microwave and optical oscillator," Opt. Lett., vol. 22, pp. 1867-1869, 1997.

[6] O. Okusaga, E. J. Adles, E. C. Levy, W. Zhou, G. M. Carter, C. R. Menyuk, and M. Horowitz, "Spurious mode reduction in dual 
injection-locked optoelectronic oscillators," Opt. Exp., vol. 19, pp. 5839_ $5854,2011$.

[7] D. Strekalov, D. Aveline, N. Yu, R. Thompson, A. B. Matsko, and L. Maleki, "Stabilizing an optoelectronic microwave oscillator with photonic filters," IEEE J. Lightw. Technol., vol. 21, no. 12, pp. 3052-3061, Dec. 2003.

[8] P. S. Devgan, V. J. Urick, J. F. Diehl, and K. J. Williams, "Improvement in the phase noise of a $10 \mathrm{GHz}$ optoelectronic oscillator using all-photonic gain," IEEE J. Lightw. Technol., vol. 27, no. 15, pp. 3189-3193, Aug. 2009.

[9] W. Li and J. Yao, "An optically tunable optoelectronic oscillator," IEEE J. Lightw. Technol., vol. 28, no. 18, pp. 2640-2645, Sep. 2010.

[10] J. Lasri, P. Devgan, R. Tang, and P. Kumar, "Self-starting optoelectronic oscillator for generating ultra-low jitter high-rate (10 GHz or higher) optical pulses," Opt. Exp., vol. 11, pp. 1430-1435, 2003.

[11] N. Yu, E. Salik, and L. Maleki, "Ultralow-noise mode-locked laser with coupled optoelectronic oscillator configuration," Opt. Lett., vol. 30, pp. 1231-1233, 2005.

[12] A. B. Matsko, L. Maleki, A. A. Savchenkov, and V. S. Illchenko, "Whispering gallery mode based optoelectronic microwave oscillator," J. Mod. Opt., vol. 50, pp. 2523-2542, 2003.

[13] A. A. Savchenkov, V. S. Ilchenko, J. Byrd, W. Liang, D. Eliyahu, A. B. Matsko, D. Seidel, and L. Maleki, "Whispering-gallery mode based opto-electronic oscillators," in Proc. IEEE Int. Freq. Contr. Symp., Jun. 2010, pp. 554-557.

[14] V. S. Ilchenko and A. B. Matsko, "Optical resonators with whisperinggallery modes-Part II: Applications," IEEE J. Sel. Topics Quantum Electron., vol. 12, no. 1, pp. 15-32, Jan./Feb. 2006.

[15] P.-H. Merrer, K. Saleh, O. Llopis, S. Berneschi, F. Cosi, and G. Nunzi Conti, "Characterization technique of optical whispering gallery mode resonators in the microwave frequency domain for optoelectronic oscillators," Appl. Opt., vol. 51, pp. 4742-4748, 2012.

[16] A. B. Matsko and V. S. Ilchenko, "Optical resonators with whisperinggallery modes-Part I: Basics," IEEE J. Sel. Topics Quantum Electron., vol. 12, no. 1, pp. 3-14, Jan./Feb. 2006.

[17] A. Chiasera, Y. Dumeige, P. Féron, M. Ferrari, Y. Jestin, G. Nunzi Conti, S. Pelli, S. Soria, and G. C. Righini, "Spherical whispering-gallery-mode microresonators," Laser Photon. Rev., vol. 51, pp. 457-482, 2010.

[18] A. A. Savchenkov, A. B. Matsko, V. S. Ilchenko, and L. Maleki, "Optical resonators with ten million finesse," Opt. Exp., vol. 15, pp. 6768-6773, 2007.

[19] K. Volyanskiy, P. Salzenstein, H. Tavernier, M. Pogurmirskiy, Y. K. Chembo, and L. Larger, "Compact optoelectronic microwave oscillators using ultra-high $Q$ whispering gallery mode disk-resonators and phase modulation," Opt. Exp., vol. 18, pp. 22358-22363, 2010.

[20] L. Larger, "Complexity in electro-optic delay dynamics: Modeling, design, and applications," Proc. R. Soc. A, to be published.

[21] Y. K. Chembo, L. Larger, H. Tavernier, R. Bendoula, E. Rubiola, and P. Colet, "Dynamic instabilities of microwaves generated with optoelectronic oscillators," Opt. Lett., vol. 32, pp. 2571-2573, 2007.

[22] Y. K. Chembo, L. Larger, and P. Colet, "Nonlinear dynamics and spectral stability of optoelectronic microwave oscillators," IEEE J. Quantum Electron., vol. 44, no. 9, pp. 858-866, Sep. 2008

[23] Y. K. Chembo, L. Larger, R. Bendoula, and P. Colet, "Effects of gain and bandwidth on the multimode behavior of optoelectronic microwave oscillators," Opt. Exp., vol. 16, pp. 9067-9072, 2008.

[24] Y. K. Chembo, K. Volyanskiy, L. Larger, E. Rubiola, and P. Colet, "Determination of phase noise spectra in optoelectronic microwave oscillators: A Langevin approach," IEEE J. Quantum Electron., vol. 45, no. 2, pp. 178186, Feb. 2009.

[25] K. Volyanskiy, Y. K. Chembo, L. Larger, and E. Rubiola, "Contribution of laser frequency and power fluctuations to the microwave phase noise of optoelectronic oscillators," IEEE J. Lightw. Technol., vol. 28, no. 18, pp. 2730-2735, Sep. 2010.

[26] R. M. Nguimdo, Y. K. Chembo, P. Colet, and L. Larger, "On the phase noise performance of nonlinear double-loop optoelectronic microwave oscillators," IEEE J. Quantum Electron., vol. 48, no. 11, pp. 1415-1423, Nov. 2012.

[27] Y. K. Chembo, A. Hmima, P.-A. Lacourt, L. Larger, and J. M. Dudley, "Generation of ultralow jitter optical pulses using optoelectronic oscillators with time-lens soliton-assisted compression," IEEE J. Lightw. Technol., vol. 27, no. 22, pp. 5160-5167, Nov. 2009.

[28] H. A. Haus, Waves and Fields in Optoelectronics. Englewood Cliffs, NJ, USA: Prentice-Hall, 1984.
[29] Y. Dumeige, S. Trebaol, L. Ghisa, T. K. Ngan Nguyen, H. Tavernier, and P. Féron, "Determination of coupling regime of high-Q resonators and optical gain of highly selective amplifiers," J. Opt. Soc. Amer. B, vol. 25, pp. 2073-2080, 2008.

[30] K. Saleh, P.-H. Merrer, O. Llopis, and G. Cibiel, "Optical scattering noise in high Q fiber ring resonators and its effect on optoelectronic oscillator phase noise," Opt. Lett., vol. 37, pp. 518-520, 2012.

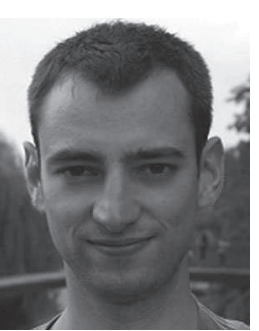

Aurélien Coillet received the M.S. degree in physics from the École Normale Supérieure de Lyon, Lyon, France, in 2008, and the Ph.D. degree in glass optical microfibers and their interest in nonlinear optics from the Laboratoire Interdisciplinaire Carnot de Bourgogne, University of Burgundy, Dijon, France, in 2011 .

Since 2012, he has been a Postdoctoral Fellow at the Franche-Comté Électronique, Mécanique, Thermique et Optique -Sciences et Technologies (FEMTO-ST) Institute, Besançon, France. His research interests include ultra-high Q whispering-gallery mode resonators, optoelectronics, nonlinear optics, and nonlinear dynamics.

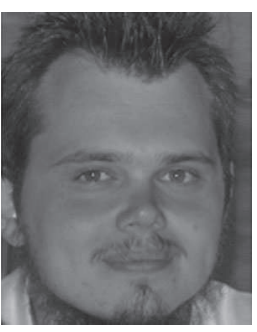

Rémi Henriet received the M.Sc. degree in physics from the University of Franche-Comté, Besançon, France, in 2011. Since 2011, he has been working toward the Ph.D. degree in opto-electronic oscillators based on whispering-gallery mode resonators at the Franche-Comté Électronique, Mécanique, Thermique et Optique -Sciences et Technologies (FEMTO-ST) Institute, Besançon, France.

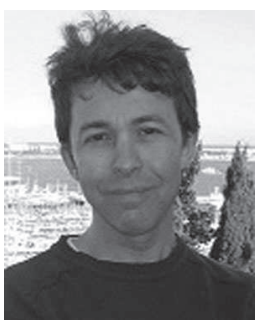

Patrice Salzenstein was born in France in 1970 He received the Master and Engineering Dipl. from the Ecole Universitaire d'ingénieurs de Lille, Lille, France, in 1993, and the Ph.D. degree in electronics from the Université des Sciences et Technologies de Lille, Lille, in 1996.

Between 1996 and 2001, he worked on microwaves applications at Thomson-CSF, Alcatel, and LCIE private research laboratories. Since 2001, he has been with the Centre National de la Recherche Scientifique (CNRS), Franche-Comté Électronique, Mécanique, Thermique et Optique-Sciences et Technologies (FEMTO-ST) Institute, Besançon, France. He managed a calibration laboratory (phase noise and short term stability of frequency) between 2002 and 2012. As a CNRS Senior Research Engineer, he is currently involved in research in optoelectronic oscillators for microwave photonics applications.

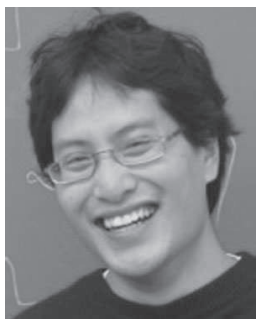

Kien Phan Huy was born on August 24, 1979 in France. He received the Ph.D. degree in optoelectronic from the Institut National Polytechnique de Grenoble, Grenoble, France.

In 2007, he joined at the Université de FrancheComté and the Franche-Comté Électronique, Mécanique, Thermique et Optique-Sciences et Technologies (FEMTO-ST) Institute, Besançon, France, as an Associate Professor. His research interests include whispering-gallery mode micro-resonators, photo-refractive solitons, pyrolitons, two-photon sources, and frequency bin entanglement. 


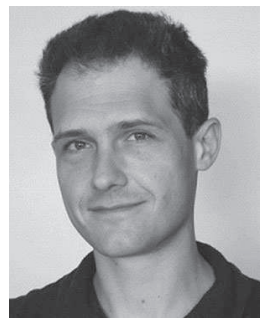

Laurent Larger (SM'11) received the Degree in electronic engineering from the University of Paris XI, Orsay, France, in 1988, the Agrégation degree in applied physics from Ecole Normale Supérieure de Cachan, Cachan, France, in 1991, and the Ph.D. degree in optical engineering and the Habilitation degree from the University of Franche-Comté, Besançon, France, in 1997 and 2002, respectively.

He was In-charge of the International Research Center GTL-CNRS Telecom, a joint laboratory between the French Centre National de la Recherche Scientifique, Georgia Tech University, Atlanta, USA, and the University of Franche-Comté, Besançon, from 2003 to 2006. He became a Full Professor at the University of Franche-Comté in 2005. He is involved in research with the Franche-Comté Electronique, Mécanique, Thermique et Optique -Sciences et Technologies (FEMTO-ST) Institute, Besançon. His current research interests include the study of chaos in optical and electronic systems for secure communications, delayed nonlinear dynamics, optical telecommunication systems, high spectral purity optoelectronic oscillators, and neuromorphic photonic computing exploiting the complexity of nonlinear dynamical transients.

Dr. Larger is an Honorary Member of the Institut Universitaire de France. He has been the Deputy Director of the FEMTO-ST Research Institute, Besançon, since 2012 .

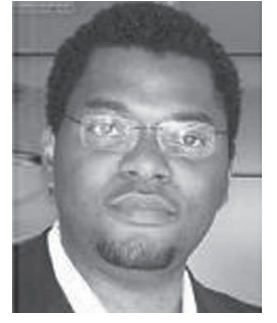

Yanne K. Chembo (SM'12) was born in France in 1976. He received the M.Sc. and Ph.D. degrees in physics from the University of Yaoundé I, Yaoundé, Cameroon, in 2001 and 2005, respectively, and the Ph.D. degree in laser physics from the Institute for Cross-Disciplinary Physics and Complex Systems, Palma de Mallorca, Spain, in 2006 .

In 2007 and 2008, he was a Postdoctoral Fellow at the Franche-Comté Électronique, Mécanique, Thermique et Optique -Sciences et Technologies (FEMTO-ST) Institute, Besançon, France. In 2009, he was awarded the National Aeronautics and Space Administration (NASA) postdoctoral program fellowship to undertake research on microwave photonic systems at the Jet Propulsion Laboratory (NASA/California Institute of Technology), Pasadena, USA. Since 2010, he has been a Senior Research Scientist at the Centre National de la Recherche Scientifique, with affiliation at the FEMTOST Institute, where he is leading the microwave photonics group. His research interests include microwave photonics, optoelectronics, semiconductor laser physics, applied nonlinear, and stochastic dynamics, information theory, and complex systems. Since 2011, he is the holder of a European Research Council (ERC) fellowship. 